\title{
RELATIONSHIP BETWEEN ELEVATED MATERNAL SERUM PODOCALYXIN CONCENTRATIONS WITH BLOOD PRESSURE VALUES AND ROUTINE LABORATORY PARAMETERS IN PREECLAMPSIA
}

\author{
Nikola K. Popovski', \\ Asparuh G. Nikolov², \\ Yordan D. Popov1, \\ Svetla O. Blazheva ${ }^{3}$
}

Clinic of Obstetrics and Gynecology, University Hospital - Pleven

${ }^{1}$ Department of Obstetrics and Gynaecology, Medical University - Pleven

${ }^{2}$ Division of Medicine, Cardiovascular Research Working Group, Institute for Scientific Research, Medical University - Pleven ${ }^{3}$ Department of Clinical Laboratory, Clinical Immunology and Allergology, Medical University - Pleven

Corresponding Author:

Asparuh Nikolov

Division of Medicine,

Cardiovascular Research Working Group,

Institute for Scientific Research,

Medical University - Pleven

1, St. Kliment Ohridski Str.

Pleven, 5800

e-mail:a_nicoloff@yahoo.com

Received: May 11, 2021

Revision received: June 03, 2021

Accepted: November 25, 2021

\section{Summary}

Podocalyxin (PCX) is a glycoprotein member of the CD34 transmembrane sialomucin family and covers the surface of podocytes. It is assumed to be a marker of glomerular endothelial injury. In conditions, podocalyxin excretion in the urine is increased as a result of podocyte injury. Recent studies show that PCX is expressed not only in kidneys but also in the endothelial cells of other organs. Preeclampsia (PE) is characterized by new-onset hypertension, generalized endothelial injury, and glomerular alteration. Having this in mind, in the present study, we aimed to: (1) determine circulating PCX levels in sera of women with preeclampsia and normal pregnancy; (2) explore for an association between PCX and blood pressure and (3) investigate a possible relationship between PCX and routine laboratory markers of cardiac and renal injury/dysfunction. Fifty-five women with preeclampsia were examined. The mean age of patients was $24.9 \pm 6$ years, and the mean age of the control group of 35 women with normal pregnancies was $24.7 \pm 5.4$ years. The enzyme-linked immunosorbent assay (ELISA) was used to determine concentrations of PCX. Levels of serum PCX in preeclamptic women were statistically significantly higher than those in women with normal pregnancy: $2.66 \pm 0.67$ vs. $2.40 \pm 0.33 \mathrm{ng} / \mathrm{ml}(\mathrm{p}=0.03)$. Podocalyxin correlated with SBP $(r=0.30 ; p=0.004)$, DBP $(r=0.35 ; \mathrm{p}=0.0007)$, uric acid $(\mathrm{r}=0.32 ; \mathrm{p}=0.002), \mathrm{CPK}(\mathrm{r}=0.22 ; 0.03)$ and its isoenzyme CK-MB $(r=0.21 ; p=0.04)$. Our results showed significantly higher levels of serum PCX in women with preeclampsia than in healthy pregnancy. Elevated podocalyxin levels are associated with an increase in blood pressure in preeclampsia. We found a relationship between PCX and routine laboratory indicators of cardiac (CPK and CK-MB) and renal injury/ dysfunction (uric acid). PCX has the potential as a future preeclampsia diagnostic biomarker.

Keywords: podocalyxin, blood pressure, preeclampsia, laboratory markers of cardiac and renal injury/dysfunction

\section{Introduction}

Preeclampsia (PE) is defined by the occurrence of new-onset hypertension $(140 / 90 \mathrm{mmHg}$ ) and either proteinuria $(0.3 \mathrm{~g}$ in a $24 \mathrm{~h}$ urine sample) or end-organ dysfunction developing after 20 weeks of gestation. $\mathrm{PE}$ occurs as a complication in approximately $2-8 \%$ of all pregnancies throughout the world [1]. It is one of the leading causes of maternal and perinatal morbidity and mortality [2]. Current approaches to preeclampsia 
propose that it involves an abnormal hemodynamic state, including diminished plasma renal flow, decreased glomerular filtration rate, and constriction of renal arteries [3,4].

Podocytes are glomerular visceral epithelial cells. They are known to be highly specialized structures [5]. Their primary function is to stabilize glomerular capillaries and to participate in the glomerular filtration barrier function. It has been reported that podocyte damage occurs in preeclampsia. These findings are supported by the abnormal shedding of podocytes in the urine [6].

Podocalyxin is a sialoglycoprotein firstly found on podocyte surfaces [7]. It was later discovered that PCX is widely expressed on the surface of endothelial cells throughout the human body $[8,9]$. PCX increases its urinary excretion in cases of glomerular endothelial damage $[10,11]$. In reports from animal studies, podocalyxin is found to be expressed in endothelial cells of many other organs like the heart, lung, and kidneys $[8,12,13]$.

Urinary excretion of podocalyxin in preeclampsia has been an object of many studies $[4,7,12,15,16]$. Interestingly, data about maternal circulatory PCX in preeclampsia are still insufficient [17-20]. Since preeclampsia is a systemic disease with generalized endothelial cells injury/dysfunction and multi-organ involvement, our primary goal was to determine serum podocalyxin concentrations in patients

Table 1. Clinical data of women with preeclampsia and normal pregnant women

\begin{tabular}{|c|c|c|c|}
\hline & Normal pregnant women & Preeclampsia & $\mathrm{P}$ \\
\hline Maternal age & $24.7 \pm 5.4$ & $24.9 \pm 6$ & $\mathrm{p}>0.05$ \\
\hline$\overline{\text { BMI }}$ & $26.7 \pm 4.2$ & $34 \pm 7.3^{*}$ & $\mathrm{p}<0.001^{*}$ \\
\hline Gravida & $2(2) * *$ & $2(2)^{* *}$ & \\
\hline Parity & $1(2) * *$ & $1(2) * *$ & \\
\hline SBP (mmHg) & $116.1 \pm 9.55$ & $157.8 \pm 22 *$ & $\mathrm{p}<0.001^{*}$ \\
\hline DBP (mmHg) & $75.3 \pm 7.76$ & $100.5 \pm 10^{*}$ & $\mathrm{p}<0.001^{*}$ \\
\hline $\begin{array}{l}\text { Past history of PE } \\
\text { Pat }\end{array}$ & $0 / 35$ & $23 / 55$ & \\
\hline Family history of AH & $1 / 35$ & $26 / 55$ & \\
\hline AH before pregnancy & $0 / 35$ & $15 / 55$ & \\
\hline Umbilical artery PI & $1.09 \pm 0.02$ & $1.24 \pm 0.12 *$ & $\mathrm{P}=0.02$ \\
\hline Uterine artery PI & $0.79 \pm 0.12$ & $1.19 \pm 0.44 *$ & $\mathrm{P}<0.001 *$ \\
\hline$\overline{\mathrm{PP}}$ & $40.8 \pm 7.32$ & $57.3 \pm 16.1^{*}$ & $\mathrm{p}<0.001^{*}$ \\
\hline$\overline{\mathrm{MAP}}$ & $88.8 \pm 7.69$ & $119.7 \pm 13.1^{*}$ & $\mathrm{p}<0.001^{*}$ \\
\hline$\overline{\text { Urea }}$ & $2.96 \pm 0.78$ & $3.75 \pm 1.63^{*}$ & $\mathrm{p}=0.01^{*}$ \\
\hline Creatinine & $75.78 \pm 14.45$ & $73.33 \pm 15.33$ & $\mathrm{p}>0.05$ \\
\hline Uric acid & $205.6 \pm 40.2$ & $326.8 \pm 105.93^{*}$ & $\mathrm{p}<0.001^{*}$ \\
\hline Total protein & $68.89 \pm 3.16$ & $58.71 \pm 8.78^{*}$ & $\mathrm{p}<0.01^{*}$ \\
\hline Albumin & $37.31 \pm 2.78$ & $31.67 \pm 4.98^{*}$ & $\mathrm{p}<0.01^{*}$ \\
\hline$\overline{\text { ASAT }}$ & $8.43 \pm 2.33$ & $20.67 \pm 7.82^{*}$ & $\mathrm{p}<0.01^{*}$ \\
\hline$\overline{\text { ALAT }}$ & $9.83 \pm 2.50$ & $27.76 \pm 8.25^{*}$ & $\mathrm{p}<0.01^{*}$ \\
\hline$\overline{\text { LDH }}$ & $369 \pm 70.78$ & $435.25 \pm 80.74^{*}$ & $\mathrm{P}=0.04 *$ \\
\hline$\overline{\text { PLT }}$ & $237.26 \pm 61.12$ & $228.74 \pm 88.53$ & $\mathrm{p}>0.05$ \\
\hline Podocalyxin & $2.40 \pm 0.33$ & $2.66 \pm 0.67^{*}$ & $\mathrm{P}=0.03^{*}$ \\
\hline$\overline{\mathrm{CPK}}$ & $83.1 \pm 23.77$ & $130.5 \pm 46.8^{*}$ & $\mathrm{P}<0.05^{*}$ \\
\hline CK-MB & $15.3 \pm 3.3$ & $24.3 \pm 7.9^{*}$ & $\mathrm{P}<0.05^{*}$ \\
\hline Number & $(\mathrm{n}=35)$ & $(n=55)$ & \\
\hline
\end{tabular}

Data are shown as the mean $\pm \mathrm{SD}$;

$* \mathrm{p}<0.05 ; * *$ Data are expressed as median (interquartile range) 
with preeclampsia and women with normal pregnancies. We also aimed to investigate whether PCX levels are associated with blood pressure values and routine laboratory parameters of cardiac and renal injury/dysfunction.

\section{Materials and Methods}

The study was approved by the Ethics Committee of Medical University - Pleven. All participants signed informed consent. Study procedures followed all guidelines for ethical standards of the responsible committee on human experimentation and the Helsinki Declaration of 1975, as revised in 2000. All patients were residing in the Clinic of Obstetrics and Gynecology of Georgi Stranski University Hospital in Pleven. We collected sera of subjects from October 2019 to March 2021. The study group consisted of 55 women with preeclampsia. The mean age of the patients was $24.9 \pm 6$ years, and that of the 35 controls with normal pregnancies was $24.7 \pm 5.4$ years. (Table 1).

The inclusion criteria for the study were as follows: 1) pregnant women with clinical symptoms and laboratory criteria for preeclampsia; maintaining a current diet and exercise during the study; 2) signed informed consent to participate in the study; 3) dysfunction of a mother's organ such as HELLP syndrome, renal failure, neurological involvement, hepatic involvement, and fetal growth retardation. Criteria for exclusion from the study were diabetes mellitus, kidney and heart disease, signs of chorioamnionitis, and the presence of a fetus with a chromosomal abnormality.

Enzyme-linked immunosorbent assay (ELISA) was used to determine Podocalyxin levels. According to the manufacturer's instructions, PCX was measured in serum samples using an ELISA kit (Human Podocalyxin ELISA kit- Reagent Genie).

Statistical analysis was performed using the following computer programs to analyze the research data: Excel (Microsoft Corporation, Redmond, WA) and Statgraphics Plus (Manugistics, Rockville, MD) for Windows. Tables, graphs, numerical values (mean $\pm \mathrm{SD}$, share indicators, and correlations) were used to describe all results. The level of significance was determined as $\mathrm{p}<0.05$. Median $(\mathrm{M})$ value was used in cases different from a normal distribution, with first and third quartile Q1 and Q3; (twentyfifth and seventy-fifth percentile P25 and 75P). Linear regression analysis was carried out to confirm the existence of a significant relationship between the variables.

\section{Results}

Levels of serum PCX in preeclamptic women were statistically significantly higher than those in women with normal pregnancy: $2.66 \pm 0.67$ vs. $2.40 \pm 0.33 \mathrm{ng} / \mathrm{ml} \quad(\mathrm{p}=0.03)$ (Figure 1). Podocalyxin levels correlated with systolic blood pressure $(\mathrm{SBP})(\mathrm{r}=0.30 ; \mathrm{p}=0.004)$, diastolic blood

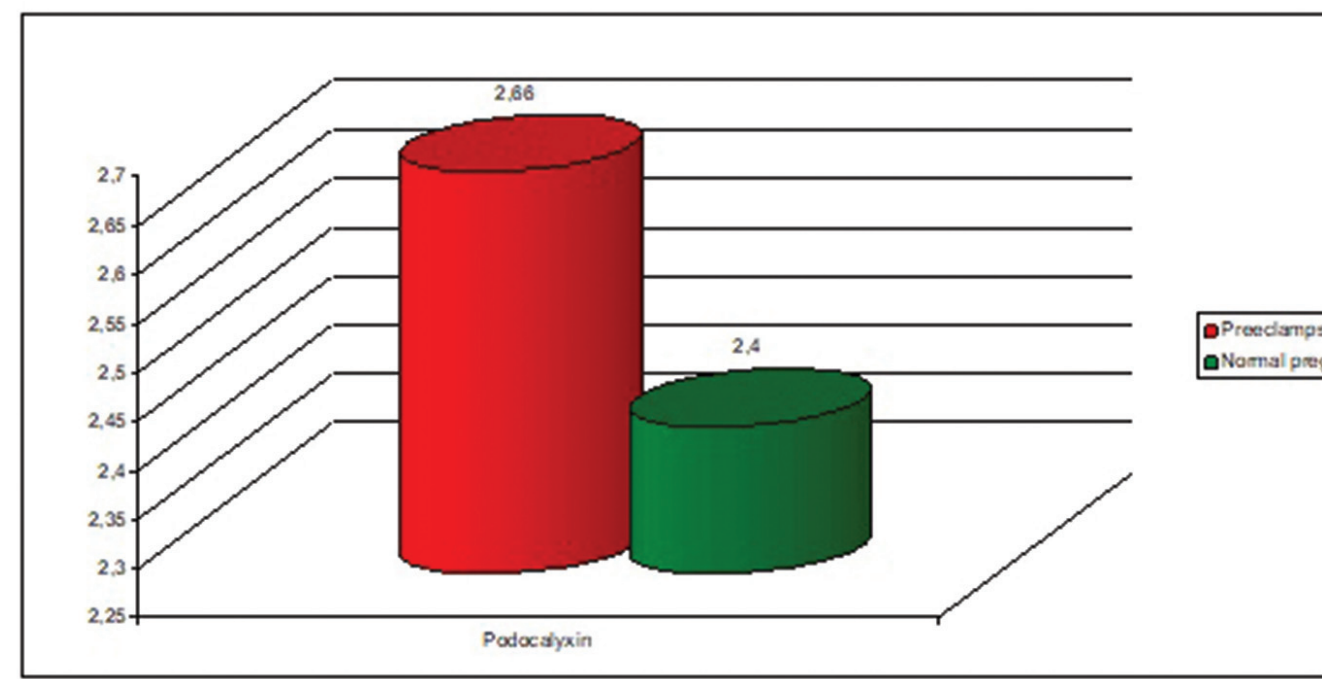

Figure 1. Serum Podocalyxin levels in preeclampsia and normal pregnant women

Serum levels of PCX in sera of women with preeclampsia were statistically significantly higher: $2.66 \pm 0.67$ vs. $2.40 \pm 0.33 \mathrm{ng} / \mathrm{ml}(\mathrm{p}=0.03)$ 


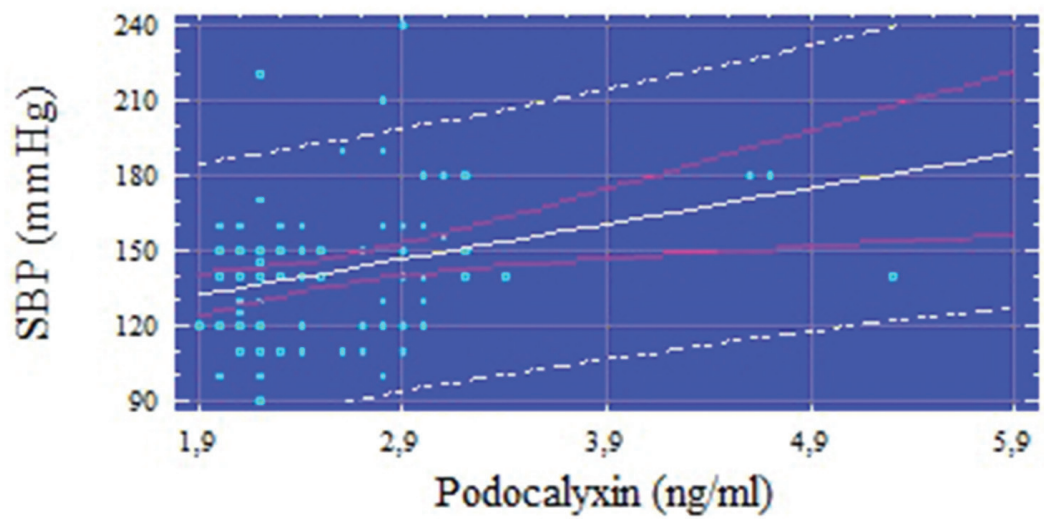

Figure 2. Linear regression analysis, showing the results of fitting a linear model to describe the relationship between Podocalyxin and SBP

There is a statistically significant relationship between Podocalyxin and SBP at 99\% confident interval SBP $(\mathrm{r}=0.30 ; \mathrm{p}=0.004)$

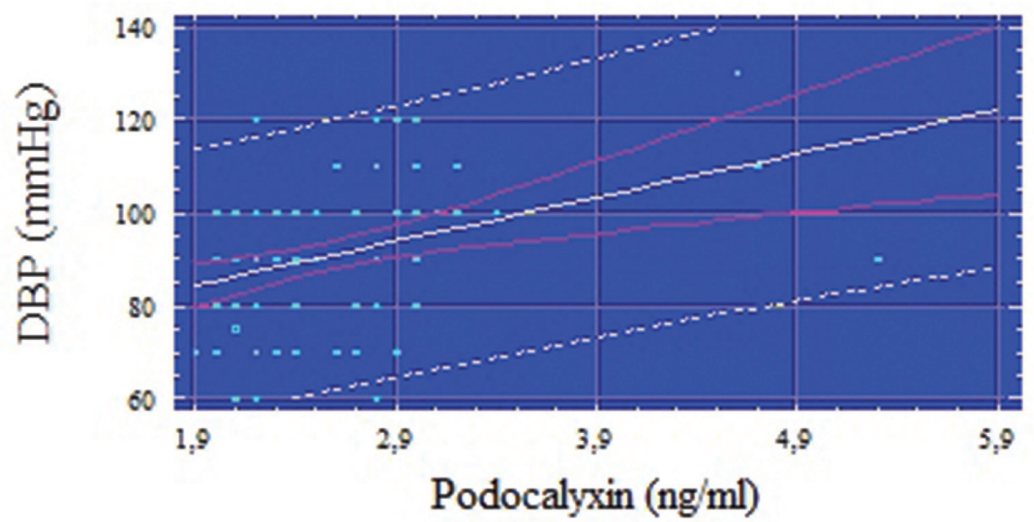

Figure 3. Linear regression analysis, showing the results of fitting a linear model to describe the relationship between Podocalyxin and DBP

There is a statistically significant relationship between Podocalyxin and DBP at $99 \%$ confident interval DBP $(\mathrm{r}=0.35 ; \mathrm{p}=0.0007)$

pressure $(\mathrm{DBP})(\mathrm{r}=0.35 ; \mathrm{p}=0.0007)$, uric acid $(\mathrm{r}=0.32 ; \mathrm{p}=0.002)$, CPK $(\mathrm{r}=0.22 ; 0.03)$ and $\mathrm{CK}-$ $\mathrm{MB}(\mathrm{r}=0.21 ; \mathrm{p}=0.04)$. Linear regression analysis confirmed the results of fitting a linear model to describe the relationship between Podocalyxin and SBP and DBP (Figure 2 and Figure 3).

\section{Discussion}

Preeclampsia is one of the most common pregnancy disorders. It is defined by the occurrence of new-onset hypertension (140/90 $\mathrm{mmHg})$ and either proteinuria $(0.3 \mathrm{~g}$ in a $24 \mathrm{~h}$ urine sample) or end-organ dysfunction developing after 20 weeks of gestation. PE is one of the leading causes of maternal and perinatal morbidity and mortality. To assess the stratification of risk and prevent further complications, early identification of PE is crucial. However, it has not been thoroughly explored.

Podocalyxin is a glycoprotein found not only on the surface of podocytes. It is also expressed on the endothelial surface throughout the human body. Based on current understandings, preeclampsia is a systemic disease characterized by hypertension and signs of damage to another organ system. It most often involves alteration of the liver or kidneys with or without proteinuria. There is growing evidence for endothelial involvement in the pathophysiology of preeclampsia. Studies have shown that podocalyxin is likely to be released into the circulation during pregnancy in association with vessel remodeling $[8,9]$.

Few studies in the literature have explored maternal serum PCX concentrations in preeclampsia to the best of our knowledge. We found statistically significantly higher serum PCX levels in preeclampsia in the study group 
than in the controls. Moreover, there were significant relationships between PCX levels and blood pressure values and between PCX and uric acid, and between creatine phosphokinase and its isoenzyme CK-MB. Of note, the levels of serum CPK and its isoenzyme CK-MB in preeclampsia were significantly higher than in normal pregnancy. However, they did not exceed the upper limit of the normal reference range. Uric acid levels were significantly increased in the patients with PE and exceeded the normal levels, which is understandable and results from renal tubular secretion.

In 2011, Dimitrakova and Kostov measured urinary cytokines IL-1a, IL-1b, IL-6, TNF-a levels by ELISA in pregnant women with acute pyelonephritis and 30 healthy controls. They found higher mean urinary cytokine levels in patients than in the controls. They concluded that pro-inflammatory cytokines could be used as reliable biomarkers for acute pyelonephritis in pregnant women [21]. In other studies, Dimitrakova et al. examined serum levels of placental soluble fms-like tyrosine-kinase-1 (sFlt-1) in pregnant women with preeclampsia and healthy women. In this study, the patients had mean serum sFlt-1 levels twice as high as those with normal pregnancies. This finding implied the role of sFlt-1 in the pathogenesis of preeclampsia and its potential to be a diagnostic marker of preeclampsia [22,23]. In addition, Dimitrakova et al. (2007) investigated the relationships between circulating placental angiogenic proteins and serum IL-2, IL-6, and IL-10 levels in pregnant women with preeclampsia. The authors reported that the imbalance of circulating angiogenic factors played a crucial role in the pathogenetic mechanism of endothelial dysfunction and clinical manifestations of preeclampsia [24].

Chen et al., 2017 found that PCX serum levels were significantly elevated in preeclampsia, especially the early-onset subtype. Furthermore, podocalyxin expression was reported in endothelial cells in different human organs, including the heart, brain, lung, ovary, and kidney [17]. Mansilla et al. (2018) conducted a study to explore to find out whether serum podocalyxin levels change before the clinical presentation of preeclampsia“". They investigated women at 11-13 weeks of gestation and found that podocalyxin serum concentrations were significantly elevated in women who subsequently developed preeclampsia" [18]. Üstünyurt E et al. (2020) evaluated plasma levels of podocalyxin in preeclampsia patients and women with normal pregnancies. The authors did not find significant differences when podocalyxin levels in preeclampsia and the control group were compared [19].

Our results are consistent with those reported by Chen et al., 2017 [17] and Amin et al., 2019 [20]. These studies also used ELISA techniques and serum samples from preeclampsia patients for analysis. The present research confirmed that elevated PCX levels might indicate endothelial cell injury/dysfunction. To our knowledge, this is the first study reporting relationships between maternal serum podocalyxin concentrations with blood pressure values and routine laboratory parameters in preeclampsia. These data were also validated by linear regression analysis. Considering these results, we suggest that podocalyxin be involved in the pathogenic mechanisms in the increase of blood pressure in preeclampsia and is probably a promising marker for the diagnosis of PE.

\section{Conclusion}

Serum podocalyxin concentrations are significantly higher in women with preeclampsia than in healthy pregnancy. Elevated podocalyxin levels are associated with an increase in blood pressure in preeclampsia. There is a relationship between PCX and routine laboratory indicators of cardiac (CPK and CK-MB) and renal injury/ dysfunction (uric acid). PCX has the potential as a future preeclampsia diagnostic biomarker.

\section{Acknowledgments}

This research was part of a university scientific project N1/2020 funded by Medical University Pleven, Bulgaria.

\section{References}

1. American College of Obstetricians and Gynecologists. Hypertension in pregnancy. Report of the American College of Obstetricians and Gynecologists' task force on hypertension in pregnancy. Obstet Gynecol. 2013;122(5):112230 . 
2. Eiland E, Nzerue C, Faulkner M. Preeclampsia 2012. J Pregnancy. 2012;586578.

3. Prakash J, Ganiger VC. Acute Kidney Injury in Pregnancy-specific Disorders. Indian J Nephrol. 2017;27(4):258-70.

4. Palacios de Franco Y, Velazquez K, Segovia N. Urinary podocalyxin as a marker of preeclampsia in a Hispanic population. Int $\mathrm{J}$ Physiol Pathophysiol Pharmacol. 2014;6(2):115-24.

5. Assady S. et al. New Insights into podocyte biology in glomerular health and disease. J Am Soc Nephrol. 2017;28(6):1707-15.

6. Nielsen JS, McNagny KM. The role of podocalyxin in health and disease. J Am Soc Nephrol. 2009;20(8):1669-76.

7. Goulopoulou S. Vascular endothelium: a potential source of podocalyxin in serum from pregnancies with preeclampsia. J Hypertens. 2017;35(11):2176-7.

8. Horvat R, Hovorka A, Dekan G, Poczewski $\mathrm{H}$, Kerjaschki D: Endothelial cell membranes contain podocalyxin: The major sialoprotein of visceral glomerular epithelial cells. J Cell Biol. 1986;102:484-91.

9. Sassetti C, Tangemann K, Singer MS, Kershaw DB, Rosen SD: Identification of podocalyxinlike protein as a high endothelial venule ligand for L-selectin: Parallels to CD34. J Exp Med. 1998;187:1965-75.

10. Hara M, Yamagata K, Tomino Y, et al. Urinary podocalyxin is an early marker for podocyte injury in patients with diabetes: establishment of a highly sensitive ELISA to detect urinary podocalyxin. Diabetologia. 2012;55(11):291319.

11. Camici M. Urinary detection of podocyte injury. Biomed Pharmacother. 2007;61(5):245-9.

12. Craici IM, Wagner SJ, Weissgerber TL, Grande JP, Garovic VD. Advances in the pathophysiology of preeclampsia and related podocyte injury. Kidney Int. 2014;86(2):275-85.

13. Biarc J, Simon R, Fonbonne C, Léonard JF, Gautier JC, Pasquier O, et al. Absolute quantification of podocalyxin, a potential biomarker of glomerular injury in human urine, by liquid chromatography-mass spectrometry. J Chromatogr A. 2015;1397:81-5.

14. Kwon SH, Woollard JR, Saad A, Garovic VD, Zand L, Jordan KL, et al. Elevated urinary podocyte-derived extracellular microvesicles in renovascular hypertensive patients. Nephrol Dial Transplant. 2017;32(5):800-7.

15. Martineau T, Boutin M, Côté AM, Maranda B, Bichet DG, Auray-Blais C. Tandem mass spectrometry analysis of urinary podocalyxin and podocin in the investigation of podocyturia in women with preeclampsia and Fabry disease patients. Clin Chim Acta. 2019;495:67-75.

16. Ylbe Palacios de F, Velazquez K, Segovia N, Sandoval G, Gauto E. Long term follow up of biomarkers of podocyte damage and renal function in patients with and without preeclampsia. Braz J Nephrol. 2018;40(4):33943.

17. Chen Q, Wang Y, Li Y, Zhao M, Nie G. Serum podocalyxin is significantly increased in earlyonset preeclampsia and may represent a novel marker of maternal endothelial cell dysfunction. J Hypertens. 2017;35(11):2287-94.

18. Mansilla M, Wang Y, Hyett J, da Silva Costa F, Nie G. Serum podocalyxin for early detection of preeclampsia at 11-13 weeks of gestation. Placenta. 2018;71:13-15.

19. Üstünyurt E, Çift T, Özdemir H, Temur M, Şimşek D, Budak Y. Podocalyxin levels in preeclampsia and relationship with severity of disease. Ann Clin Anal Med. 2020;11(3): S27983.

20. Amin A, Abou-Taleb H, Gamal M, Thabet M, Azoz N, Abbas A. Evaluation of Podocalyxin level in preeclampsia with severe features ${ }^{6}$ patients: a cross-sectional study. Int J Reprod Contracept Obstet Gynecol. 2019;8(8):3255-8.

21. Dimitrakova E, Kostov I. Studies on the level of pro-inflammatory cytokines IIL-1a, IL-1b, IL-6, TNF-a in pregnant women with acute pyelonephritis. Akush Ginekol. 2011;50(2):3-6.

22. Dimitrakova ED, Dimitrakov JD, Karumanchi SA, Pehlivanov BK, Milchev NP, Dimitrakov DI Placental soluble fms-like tyrosine-kinase-1 (sFlt-1) in pregnant women with preeclampsia. Folia Med. 2004;46(1):19-21.

23. Dimitrakova E, Milchev N, Dimitrakov D, Batashki I, Gŭrova A. Placental angiogenic factors. Essentials and clinical significance in preeclampsia. Akush Ginekol. 2006;45(3):52-8.

24. Dimitrakova E, Milchev N, Batashki I, Karumanchi SA. Cause-effect relatioships between circulating placental angiogenic proteins and serum IL-2, IL-6 and IL-10 levels in pregnant women with preeclampsia. Akush Ginekol. 2007;46(9):4-8. 